\title{
Density-functional simulations of lithium intercalation in rutile
}

\author{
Marina V. Koudriachova, ${ }^{1}$ Nicholas M. Harrison, ${ }^{2}$ and Simon W. de Leeuw ${ }^{1}$ \\ ${ }^{1}$ Computational Physics, Department of Applied Physics, TU Delft, Lorentzweg 1, 2628 CJ Delft, The Netherlands \\ ${ }^{2}$ Department of Chemistry, Imperial College of Science, Technology and Medicine, London, SW7 2AY, United Kingdom \\ and CLRC, Daresbury Laboratory, Daresbury, Warrington, WA4 4AD, United Kingdom
}

(Received 30 June 2001; revised manuscript received 7 February 2002; published 7 June 2002)

\begin{abstract}
Density-functional simulations of lithium intercalation into rutile structured titanium dioxide are presented. Full relaxation of structures for a wide range of insertion concentrations is used to identify the thermodynamically most stable configurations and Li-ion site preferences. The host lattice is found to undergo large deformations upon Li insertion, which can be related to the excitation of soft vibrational modes. The dominant screening interaction is found to be due to these elastic distortions of the lattice rather than to dielectric screening. This leads to highly anisotropic and concentration-dependent effective Li-Li interactions, which are not easily amenable to empirical parametrization. The anisotropic volume expansion is found to be largely due to the increase in the radii of reduced $\mathrm{Ti}$ ions as they accommodate charge donated to the lattice. The computed open circuit voltage $(\mathrm{OCV})$ reproduces the characteristic features of experimental discharge curves at elevated temperature. The computed Li-ion energy surfaces reveal highly anisotropic diffusion. A model of Li intercalation is proposed, which takes account of both the thermodynamic and kinetic properties computed here. This model is used to resolve apparent contradictions in the current interpretation of the measured OCV and its dependence on temperature. Predicted changes in the electronic structure and their relationship to the interaction between structural, charge, and spin degrees of freedom are discussed in detail.
\end{abstract}

DOI: 10.1103/PhysRevB.65.235423

PACS number(s): 66.30. $-\mathrm{h}, 64.70 . \mathrm{Kb}, 82.45 .-\mathrm{h}$

\section{INTRODUCTION}

Transition-metal oxides have attracted widespread attention because of their possible application as an (inexpensive) electrode material in all-solid-state batteries ${ }^{1,2}$ and dyesensitized solar cells. ${ }^{3}$ The main reasons for this are that the transition-metal cations are able to accommodate electron density donated by intercalated $\mathrm{Li}$ atoms and that the structures are open enough to provide space for the $\mathrm{Li}$ ions. These properties result in a number of low-energy sites for Li ions within the lattice and the potential for high capacity Li-ion intercalation. For good ionic conductivity the interstitial space in the oxide framework must be interconnected and give rise to a potential energy landscape, which is sufficiently smooth to support high Li-ion mobility. Nanostructured titanium dioxide $\left(\mathrm{TiO}_{2}\right)$ is a particularly interesting candidate as a host material because of its high surface area, chemical stability, semiconducting properties, and low cost. The most common natural form of $\mathrm{TiO}_{2}$ is rutile. Lithium intercalation in rutile has been studied extensively with a variety of experimental and theoretical techniques. Despite this work, to our best knowledge, no unified picture of the intercalation process has emerged and a number of contradictory results have been reported. For example, the maximum electrochemical insertion $x$ (the atomic ratio, Li/Ti) has been reported to have various values between 0.1 and $1,{ }^{4-9}$ which clearly depends strongly on the experimental conditions. Similarly, theoretical studies ${ }^{10-13}$ have not provided a consistent picture of the ordered structures formed or the nature of the local deformations around the Li ions. This is due, in part, to the fact that in previous simulations restrictions have been applied to geometric relaxations either by imposing rigid cluster boundary conditions ${ }^{11}$ or by considering only the isotropic variations of the unit cell. ${ }^{13}$
In the current study the results of fully relaxed periodic density-functional theory calculations of $\mathrm{Li}$ intercalation in rutile are presented. This work extends a preliminary report in which the importance of Li-ion diffusion on the performance of this system as a battery electrode was noted. ${ }^{14}$ The preferred intercalation sites, and the most stable ordered configurations for inserted ions have been analyzed for a wide range of insertion concentrations. The diffusion pathways for $\mathrm{Li}$ ions have been explored in detail and a model of $\mathrm{Li}$ intercalation is presented. The changes in electronic structure and their coupling to local distortions are also examined. The paper is organized as follows. In Sec. II the details of the calculations are described briefly. In Sec. III the structure of Li-intercalated rutile is discussed and the relative stabilities of the octahedral and tetrahedral sites are analyzed. The relationship between the local deformation induced upon $\mathrm{Li}$ insertion and the normal vibrational modes of pure rutile ${ }^{15}$ are considered in Sec. IV. In Sec. V the ordering of $\mathrm{Li}$ ions over the available insertion sites at different loading is examined. In Sec. VI the open circuit voltage (OCV) profile is computed and compared to that observed. The diffusion of $\mathrm{Li}$ ions in the host lattice and the lattice's role as a kinetic constraint are discussed in Sec. VII. Section VIII deals with the charge transfer between the intercalated $\mathrm{Li}$ and the host lattice and with the induced changes in the electronic structure. Conclusions are drawn and discussed in Sec. IX.

\section{DETAILS OF THE SIMULATIONS}

Total-energy calculations based on the plane-wave pseudopotential approach were performed using a variant of CASTEP software ${ }^{16,17}$ and supplemented by all electron calculations using CRYSTAL. ${ }^{18}$ CRYSTAL was also used to provide local orbital analysis of the charge and spin distributions and 
the electron structure. The electronic ground state was found through conjugate-gradient minimization of the total energy with respect to the plane-wave coefficients. A $380-\mathrm{eV}$ planewave cutoff was found to be sufficient to converge the total energy of a unit cell of rutile to within $0.01 \mathrm{eV}$. Electronic exchange and correlation were described using the spindependent generalized-gradient approximation to densityfunctional theory (DFT). ${ }^{19}$ In order to break the spin symmetry, spin-polarized calculations were performed under the constraint of a fixed total spin per cell and the lowest energy was sought by varying the total spin. Depending on the composition, various supercells of between one and eight rutile unit cells were used. Brillouin-zone sampling was performed on a Monkhorst-Pack grid with the density of points determined from a spacing of $0.1 \AA$. Ultrasoft pseudopotentials ${ }^{20}$ were used to replace the $\mathrm{Ti}(1 s, 2 s, 2 p), \mathrm{O}(1 s)$, and $\mathrm{Li}$ $(1 s)$ core orbitals. Explicit treatment of the semicore Ti $(3 s$, $3 p$ ) states as valence orbitals was found to be important as has previously been shown in studies of the structure of bulk rutile. ${ }^{21}$ Moreover, as is demonstrated in Sec. VIII, the shift of the semicore Ti levels depends on the insertion concentration. If this relaxation is neglected an overestimation of the cell voltages is obtained. Mechanical equilibrium was achieved by relaxation of the ionic positions and the size and shape of a computational unit through conjugate-gradient minimization of the total energy.

\section{SITE PREFERENCES}

Initially the stability of the various sites available for the guest ions in the rutile structure was examined. Rutile has a tetragonal structure with calculated lattice parameters $a=b$ $=4.63$ and $c=2.96 \AA$, which are in excellent agreement with the observed values of $a=b=4.59$ and $c=2.95 \AA .{ }^{22} \mathrm{In}$ the following all calculations are based on this minimumenergy geometry.

The rutile structure consists of $\mathrm{TiO}_{6}$ polyhedra that share edges in the $c$ direction and corners in the $a b$ planes (Fig. 1). This packing results in rutile's highly anisotropic elastic and dielectric properties. ${ }^{23}$ The two types of sites, which could in principle accommodate $\mathrm{Li}$ ions, are indicated in Fig. 1 in pink and blue spheres. The tetrahedral sites (blue) are formed by the $\mathrm{TiO}_{6}$ polyhedra sharing edges in the $c$ direction while the octahedral sites (pink) are built from corner-sharing polyhedra. A unit cell (containing $\mathrm{Ti}_{2} \mathrm{O}_{4}$ ) provides four octahedral and four tetrahedral intercalation sites.

The octahedral site is not entirely regular, having a height of $3.33 \AA$ and a rectangular base of $2.96 \times 3.33 \AA^{2}$. The tetrahedral site is a distorted pyramid with sides of 2.79 and $2.96 \AA$. Ionic radius considerations indicate that there is less space available in the tetrahedral site for guest ions and that neither site can accommodate $\mathrm{Li}$ ions without significant structural deformation involving expansion of the site in the $a b$ plane.

The ionic picture is confirmed by model calculations in which the effect of introducing a single Li atom at each of these sites in a single unit cell $\left(x=\frac{1}{2}\right)$ was examined. The inserted $\mathrm{Li}$ atom is ionized, donating charge to the lattice. In either site the position of the resultant $\mathrm{Li}$ ion is largely de-

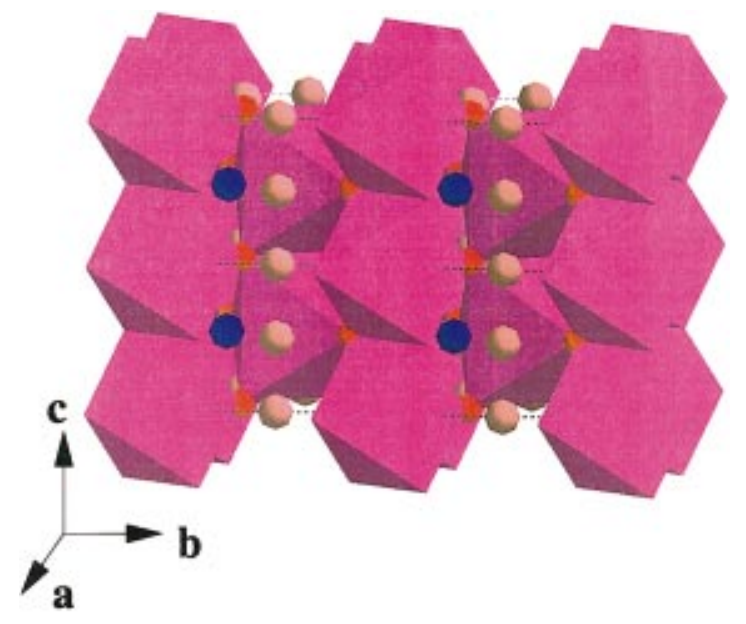

FIG. 1. (Color) A polyhedral representation of four unit cells of the structure of rutile. Two possible sites for intercalation with octahedral or tetrahedral coordination are marked in pink and blue, respectively.

termined by interaction with the nearby $\mathrm{O}$ ions. When placed in a tetrahedral site the $\mathrm{Li}$ ion adopts a position with essentially fourfold coordination. It forms two ionic bonds of 1.85 and two of $1.93 \AA$ with the neighboring O ions. As expected, incorporation of $\mathrm{Li}$ in this site results in a strong distortion of the lattice as shown in Fig. 2. The neighboring Ti ions are dislodged from their crystallographic positions moving away from the $\mathrm{Li}$ ion. This distortion results in a reduction of the lattice symmetry to monoclinic $(P 11 \mathrm{~m})$ with a cell angle between the $a$ - and $b$-lattice vectors of $\approx 80^{\circ}$ and a dramatic increase of the cell volume (by $\approx 15 \%$ ). The expansion is highly anisotropic with $8 \%$ increases in the $a$-and $b$-lattice constants while $c$ actually contracts slightly (by $1 \%$ ).

The computed energies indicated a strong preference, by $0.7 \mathrm{eV} / \mathrm{Li}$ ion, for intercalation into the octahedral site. Within a unit cell there are two symmetrically distinct local minima for $\mathrm{Li}$ ion insertion. The first position is at the center of an octahedron with $x=0.5 a, y=0$, and $z=0.5 c$. There are equivalent positions in the nearest-neighbor octahedron in the $c$ direction with $z=0$ and in the nearest-neighboring oc-

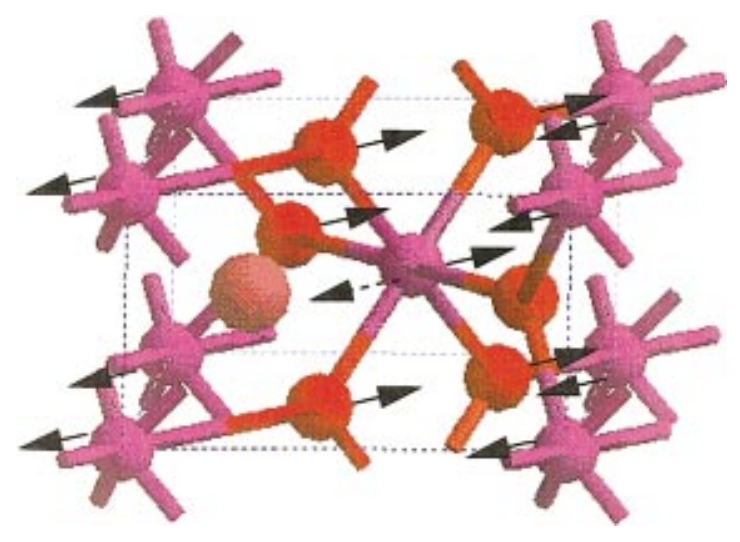

FIG. 2. (Color) The distortion induced by Li-ion intercalation in the tetrahedral site of a single cell $\left(x=\frac{1}{2}\right)$. The Li ion is in pink, $\mathrm{O}$ in red, and $\mathrm{Ti}$ in violet. The arrows indicate the direction of the induced displacements. 

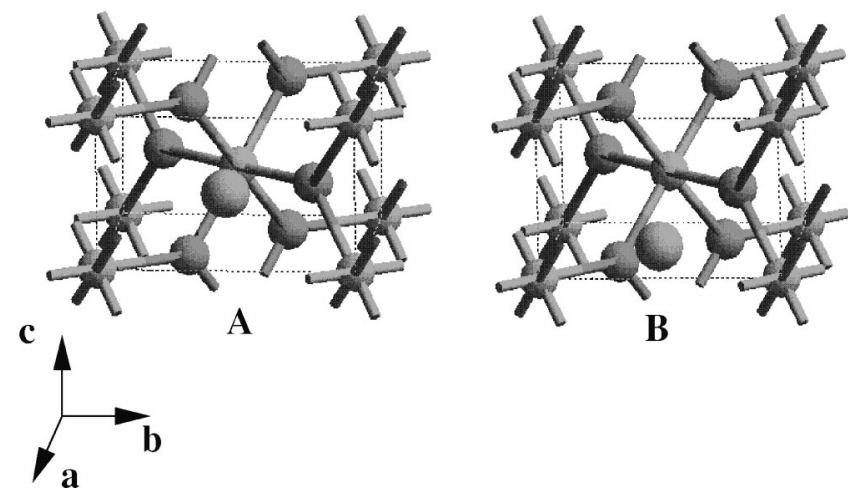

FIG. 3. The central (a) and off-center (b) positions adopted by the Li ion in an octahedral site.

tahedra in the $a b$ plane at $x=0, y=0.5 b$ with $z=0$ and $0.5 c$. The second position is off center with $z=0.2 c$ (or equivalently, $z=-0.2 c$, or $\pm 0.3 c$ ). These positions are depicted in Fig. 3. The two positions have approximately the same energy and, as is discussed below (Sec. VII), the energy barriers between them are low, so that rapid diffusion along the $c$ direction is possible. For both positions the overall structure remains close to tetragonal with $a$ and $b$ expanding by $4 \%$ and $c$ contracting slightly (by 1\%). This leads to a volume increase of $\approx 7 \%$ - significantly less than for the tetrahedral site.

The local environment of the $\mathrm{Li}$ ion is very different for the two positions within the octahedral hole. In the central position the $\mathrm{Li}$ ion remains essentially octahedrally coordinated with two neighboring oxygens at 1.87 and four at 2.08 $\AA$ A. In the off-center position the $\mathrm{Li}$ ion becomes effectively tetrahedrally coordinated. There are four neighboring $\mathrm{O}$ at $1.83-1.87$ and two at 2.57 and $2.99 \AA$.

It seems likely that the number of different low-energy positions available is determined by the size of the guest ion relative to the space available within the octahedral site. This has been explored by replacing $\mathrm{Li}^{+}$ions with $\mathrm{Na}^{+}$or $\mathrm{H}^{+}$ ions. While the larger $\mathrm{Na}^{+}$ions can occupy only one central position, the smaller $\mathrm{H}^{+}$ions find multiple low-energy positions in which $\mathrm{OH}$ bonds are formed perpendicular to the $c$ direction with a bond length of $\approx 0.98 \AA$. The $\mathrm{Li}^{+}$ion is intermediate between these two extremes. $\mathrm{Li}^{+}$interstitials in off-center positions have been observed in Li-ramsdellite structure ${ }^{24}$ in which the local structure is similar to that in rutile.

The geometry of Li-intercalated rutile has been previously considered in cluster calculations by Stashans et al. ${ }^{11}$ In their study two possible intercalation positions were found both within the tetrahedral site. The first position bisects two Ti ions on a line along the $c$ direction. Intuitively, this is a highly unfavorable position as one would expect the electrostatic repulsion of the $\mathrm{Li}$ and $\mathrm{Ti}$ ions to be poorly screened and thus prohibitively large. Examining this structure in the current study we found it to be unstable with respect to the geometry discussed above and depicted in Fig. 2. The second position considered was qualitatively similar to that proposed here but with significantly shorter Li-O and Li-Ti distances.

\section{LATTICE DEFORMATIONS AND SOFT VIBRATIONAL MODES}

An important factor in $\mathrm{Li}$ intercalation is the ease with which the host lattice can deform locally to accommodate the guest ions. The low-frequency optic modes of $\mathrm{TiO}_{2}$ rutile $\left(A_{2 u}, E_{u}, B_{1 u}, B_{1 g}\right)$ are much softer than in comparable structures such as $\mathrm{SiO}_{2}$ rutile (stishovite). For instance, the frequencies of the modes that involve atomic displacements solely along the $c$ direction $\left(A_{2 u}\right.$ and $\left.B_{1 u}\right)$ are 167-173 and $113 \mathrm{~cm}^{-1}$ in $\mathrm{TiO}_{2}$ rutile, ${ }^{25,26}$ which may be compared to 650 (Ref. 27) and $383.6 \mathrm{~cm}^{-1}$ (theoretical value ${ }^{28}$ ) in stishovite. The $A_{2 u}$ mode is a ferroelectric mode along $c$ and is related to the high static dielectric constant in this direction. $B_{1 u}$ is optically inactive but has been observed in inelastic neutronscattering experiments. ${ }^{26}$ Other soft modes $\left(B_{1 g}, E_{u}\right)$ involve atomic displacements in the $a b$ planes only. The $E_{u}$ mode is infrared active, with an observed frequency of 183 $\mathrm{cm}^{-1}$ (Ref. 26) (compared to $470 \mathrm{~cm}^{-1}$ in stishovite ${ }^{27}$ ). The $B_{1 g}$ mode is observed in Raman spectra at $142-143 \mathrm{~cm}^{-1}$ (Ref. 29) $\left(234 \mathrm{~cm}^{-1}\right.$ for stishovite $\left.{ }^{30}\right)$.

$\mathrm{Li}$ insertion into the tetrahedral site produces distortions (Fig. 2) that are similar to the excitation of the degenerate $E_{u}$ mode. It is notable that one of the neighboring Ti ions actually moves in a direction opposing that implied by the $E_{u}$ mode-as is indicated in Fig. 2 by a dashed line. This motion appears to be due to the strong $\mathrm{Li}$-Ti electrostatic repulsion, which is not effectively screened. For insertion into the octahedral site the displacements of the framework atoms correspond to a superposition (in a proportion of approximately 1:10) of the $A_{1 u}$ and $B_{2 g}$ modes for the central position, and of $A_{1 u}$ and $B_{1 u}$ for the off-center position. For both positions in the octahedral site the deformations of the host structure correspond to the displacement pattern of modes that have frequencies lower than those of the $E_{u}$ mode excited by insertion in the tetrahedral site. It appears that in addition to the greater volume available the flexibility of the octahedron strongly favors the octahedral over the tetrahedral site. In the remainder of this paper only insertion into octahedral sites will be considered.

The softness of the low-frequency modes in rutile permits the deformations required for low-energy $\mathrm{Li}$ intercalation. The much higher frequencies of the corresponding modes in other materials (stishovite, for instance) indicate that Li insertion will be thermodynamically unfavorable.

\section{Li ORDERING AT DIFFERENT CONCENTRATIONS}

It is instructive to consider an intercalation compound in terms of the lattice of sites available for guest ions. In general the ions separate under the influence of repulsive electrostatic interactions resulting in strong positional correlations instead of random placement within the lattice. At certain concentrations regular superlattices may be formed. Rapid screening of effective electrostatic repulsion means that interactions do not usually extend over more than a few lattice spacings. For high-energy density to be achieved efficient screening of these interactions without irreversible changes to the lattice structure is essential. Macroscopically 


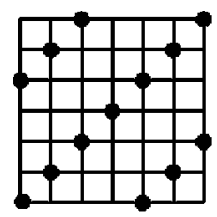

A

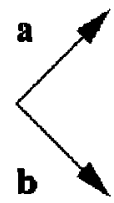

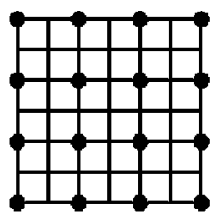

B

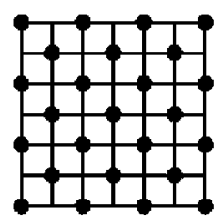

D

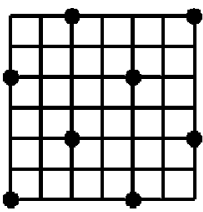

C

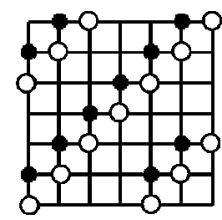

$\mathbf{E}$
FIG. 4. Ordered Li superstructures with nearest-neighbor exclusion in the $a b$ planes. The vertices of the grids correspond to unoccupied octahedra of the rutile lattice. Filled circles correspond to $z=0.5 c$, and open circles correspond to $z=0$.

the screening of an electric field is characterized by the dielectric permittivity tensor. Rutile has highly anisotropic dielectric properties: the static dielectric constant in the direction of the tetragonal axis $c$ is two times larger than that in the $a$ or $b$ directions (173 and 89, respectively ${ }^{22}$ ). This suggests that the screening of $\mathrm{Li}$-ion repulsion is more efficient in the $c$ direction. However, as will become clear below, this simple model is insufficient. The local distortions of the lattice induced by accommodation of the guest ion and the donated charge density must be considered in order to understand the effective $\mathrm{Li}-\mathrm{Li}$ interactions. The importance of elastic screening is evident from the large deformation of the local environment for the $\mathrm{Li}_{1 / 2} \mathrm{TiO}_{2}$ case discussed above.

In order to investigate these interactions, and the resulting ordering of $\mathrm{Li}$ ions, as a function of composition ordered configurations with the lowest energy were sought for $\mathrm{Li}_{x} \mathrm{TiO}_{2}$ for the concentrations $x=\left(\frac{1}{16}, \frac{1}{8}, \frac{1}{4}, \frac{1}{2}, \frac{3}{4}, 1\right)$. For each $x$ a number of periodic supercells were considered and the structures were fully relaxed. In all calculations the intercalated structures were found to be stable with respect to metallic lithium and bulk rutile.

At the lowest concentration considered here, $x=\frac{1}{16}$, the Li ions remain well separated and their effective interactions may be considered to be short range and repulsive. For higher concentrations the ordering along the $c$ direction and in the $a b$ planes must be considered. Each $a b$ plane of octahedra forms a square lattice with neighboring octahedra separated along the (110) direction by $\sqrt{2} a$. There are two such planes of octahedra per cell at $z=0$ and 0.5. Neighboring octahedra in the $c$ direction are separated by $0.5 c$.

At $x=\frac{1}{8}$ formation of Li-ion superstructures in $a b$ planes separated by at least three empty planes (one empty unit cell) in the $c$ direction is favored over structures in which octahedra in neighboring cells are occupied in the $c$ direction. The square lattice of insertion sites within an $a b$ plane is depicted in Fig. 4. For $x=\frac{1}{8}$ the lowest-energy configurations are those without nearest-neighbor occupancy in the $a b$ planes. There are only two possible configurations that allow nearest-neighbor site exclusion. The first, Fig. 4(a), consists
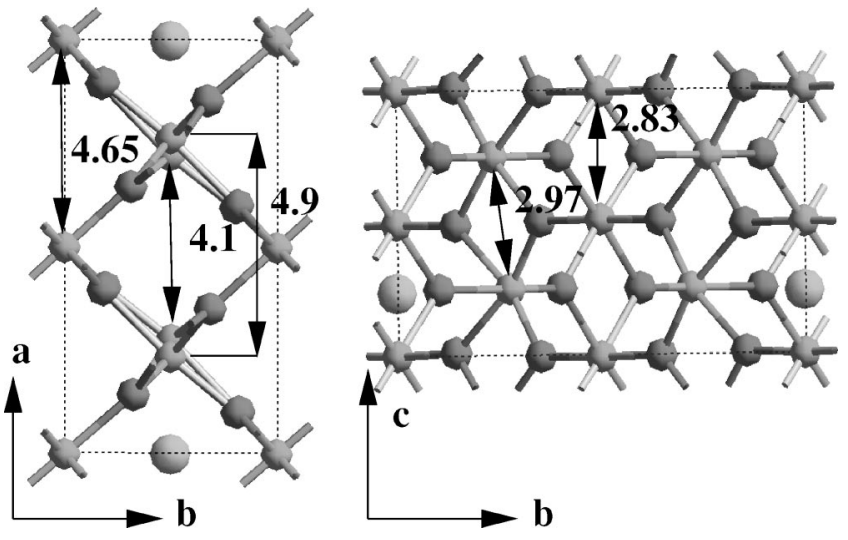

FIG. 5. The deformation of the host lattice on Li intercalation at $x=\frac{1}{8}$ for the (a) and (b) structures of Fig. 4 .

of chains along $a(b)$ that are well separated in the $b(a)$ direction. The Li ions occupy second- and fifth-neighbor lattice sites. The second configuration is the checkerboard pattern shown in Fig. 4(b). Here the Li ions occupy thirdnearest-neighbor sites. Figure 4(c) shows the configuration for $x=\frac{1}{16}$ that has an energy per inserted Li ion very similar to that of the $A$ and $B$ structures at $x=\frac{1}{8} \quad(2.69$ $\pm 0.02 \mathrm{eV} / \mathrm{Li}$ ). This establishes that in these structures the Li-ion interactions are screened very effectively and that $x$ $=\frac{1}{8}$ is representative of the dilute limit. At these concentrations the effective interactions of the $\mathrm{Li}$ ions are fully screened for occupancy of second-neighbor sites in the $a b$ planes and fourth-neighbor sites in the $c$ direction.

The screening of $\mathrm{Li}-\mathrm{Li}$ interactions is achieved through strong local deformation of the host lattice. The distortions of the neighboring octahedra in the $a b$ plane and $c$ directions for structure $A$ are displayed in Fig. 5. Neighboring Ti ions move directly away from the $\mathrm{Li}$ ion with an accompanying rotation of the neighboring $\mathrm{O}$ ions. This motion closes the nearest-neighbor octahedra in the $c$ direction. In the $a b$ planes the second-nearest-neighbor octahedra (along $a$ or $b$ ) are closed while the nearest-neighbor sites along the (111) direction are opened. The motions in the $a b$ plane are closely related to the soft $B_{1 g}, A_{1 g}$ and $E_{u}, A_{2 g}$ vibrational modes and are related to the ease with which the corner-sharing octahedra can hinge in the $a b$ planes. The local distortion in the $c$ direction corresponds predominantly to the $B_{1 u}$ vibrational mode, which is the lowest-frequency mode of the rutile lattice.

The major consequences of these distortions become apparent when the insertion concentration is increased above $\frac{1}{8}$. At this point $\mathrm{Li}$ ions begin to fill nearest-neighbor sites in the (111) direction that have been opened by the local distortions. The Li-Li interactions are best characterized as shortrange attractions along (111) and effective short-range repulsions along the $a-c$ directions. At $x=\frac{1}{4}$, the energy of the checkerboard structure [Fig. 4(d)] in which $\mathrm{Li}$ ions are separated by one site in both $a$ and $b$ directions is higher (by $0.28 \mathrm{eV}$ per $\mathrm{Li}$ ) than that of the nearest-neighbor pattern of zigzag chains exploiting the attractive interactions along the (111) direction [Fig. 4(e)].

For $x=\frac{1}{2}$ the lowest-energy structure is with $\mathrm{Li}$ ions fully 


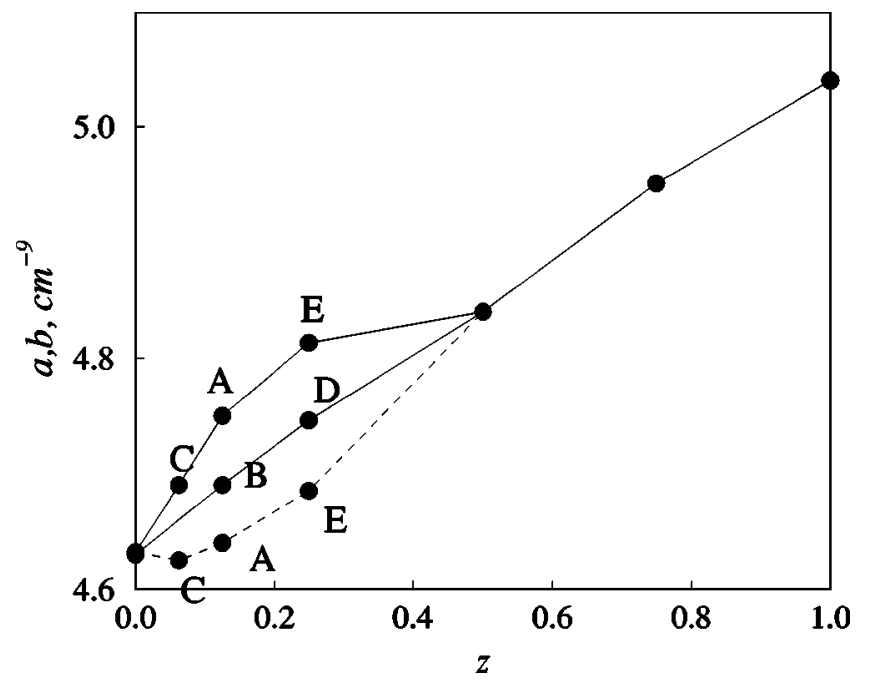

FIG. 6. The variation of the $a$ and $b$ cell parameters with $\mathrm{Li}$ concentration and ordering pattern. The solid line corresponds to $a$ and the dashed line to $b$. The labeling of the structures is defined in Fig. 4.

occupying the sites in the $a b$ planes with occupied planes separated by three empty planes in the $c$ direction. At this concentration there is considerable stress within the $a b$ planes and the Li ions move to off-center positions within the octahedra to avoid being coplanar with the Ti ions. The local distortions correspond to a superposition of the lowfrequency $B_{1 u}$ mode and the higher-frequency $A_{1 g}$ mode. None of the distortions for $x \leqslant \frac{1}{2}$ correspond to the soft mode responsible for the high static dielectric constant in the $c$ direction $\left(A_{2 u}\right)$, indicating that at these concentrations elastic screening predominates over dielectric screening.

In Fig. 6 the variation of the $a$ - and $b$-lattice constants for the various superstructures discussed above is depicted. The volume of the unit cell (up to $x=\frac{1}{2}$ ) and the average of $a$ and $b$ (up to $x=1$ ) increase linearly with Li concentration while $c$ decreases slightly. The change in volume with respect to pure rutile is considerable: around $8 \%$ at $x=\frac{1}{2}$ and $15 \%$ at $x=1$. The $a$ - and $b$-lattice constants increase by $4 \%$ at $x$ $=\frac{1}{2}$ and $8 \%$ at $x=1$, while $c$ decreases by about $1.5 \%$ as the concentration rises from 0 to 1 . In previous periodic HartreeFock calculations a volume change for $\mathrm{LiTiO}_{2}(x=1)$ of $24 \%$ was reported. ${ }^{13}$ However in these calculations only isotropic variations of the unit cell were considered, ${ }^{13}$ which, as is clear from the data reported here, are not good approximations for this system.

The linear change of volume for $x \leqslant \frac{1}{2}$ can be explained completely in terms of expansion of individual octahedral sites. At higher concentrations the volume of the unit cell expands faster than the size of the Li-containing octahedra. This indicates that the packing of the octahedra is disrupted. It seems highly likely that as the structure is distorted in this way a phase transition to an alternative structure, which reduces the local stresses, will occur. Electrochemical experiments have indicated such a transition to an unidentified structure for $x$ in the range $\frac{1}{2}-\frac{3}{4},{ }^{9}$ which is followed by a transition to a hexagonal structure at $x=0.8 .^{31}$ Comparison of the calculated and measured x-ray-diffraction (XRD) pat-

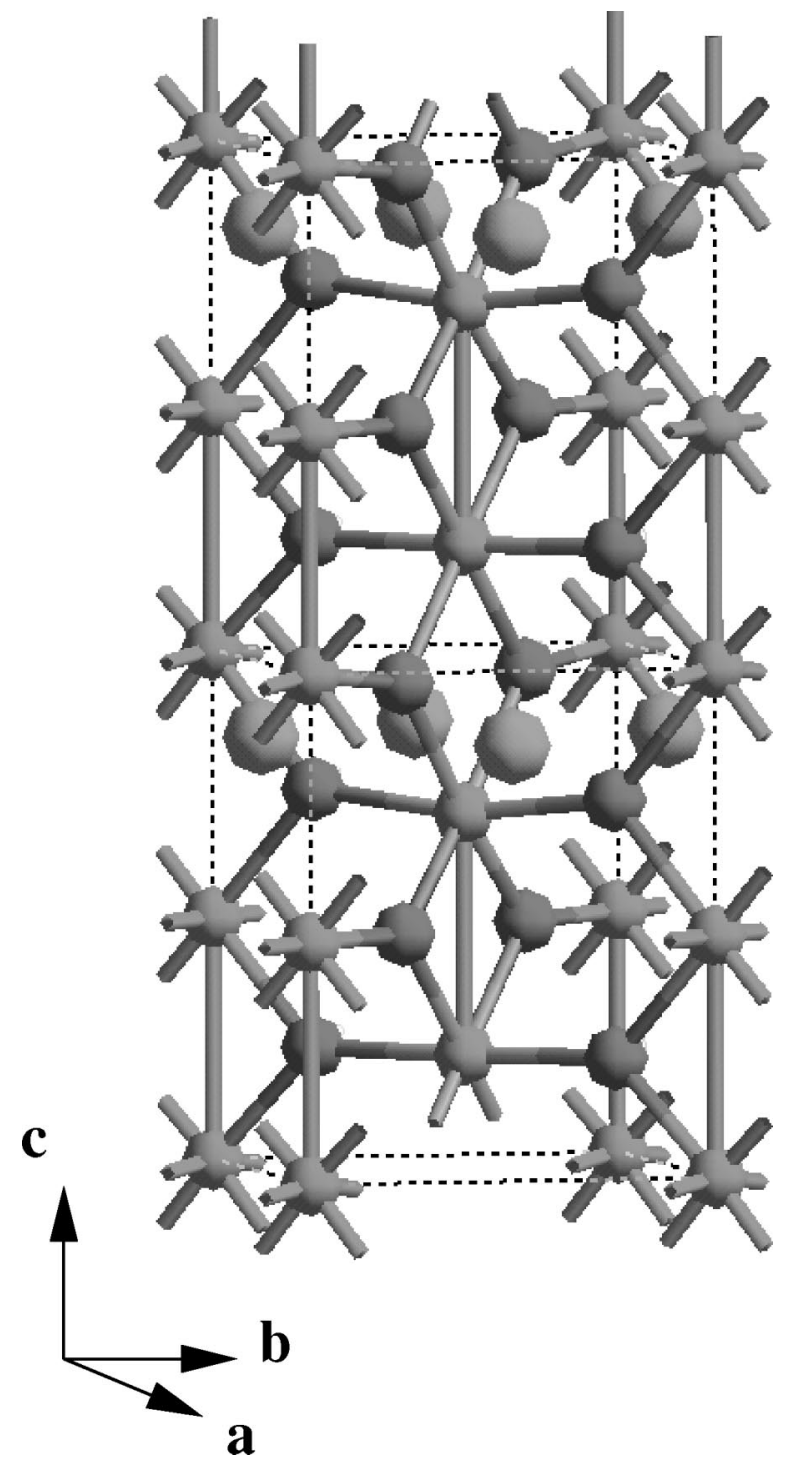

FIG. 7. The predicted layered structure at $x=\frac{1}{2}$. A Ti-Ti bond is drawn for interlayer Ti-Ti contacts closer than $2.9 \AA$.

tern indicates that the previously unidentified phase is that predicted here for $x=\frac{1}{2}$ and $\frac{3}{4}$, which is shown in Fig. $7 .^{32}$ Experimentally several other polymorphs of $\mathrm{Li}_{0.5} \mathrm{TiO}_{2}$ are known. The most stable adopts a spinel structure. The calculated cell parameters $(a=b=c=8.41 \AA)$ and XRD pattern of the spinel phase are in good agreement with the experimental data $(a=b=c=8.41 \AA) .{ }^{33}$ Although the spinel phase is computed to be $0.25 \mathrm{eV}$ per formula unit more stable it is clear that it is the metastable lithiated rutile phase predicted here that is adopted by the cathode in electrochemical experiments. $^{9}$

Full optimization of the geometry of the hexagonal structure $(x=1)$ gives cell parameters and ionic positions in excellent agreement (within less than 1\%) with both the observed and previous computed values. ${ }^{10}$ The hexagonal structure is $0.26 \mathrm{eV}$ per formula unit more stable than the fully lithiated rutile structure $(x=1)$, in agreement with its observed stability. 


\section{THE OPEN CIRCUIT VOLTAGE PROFILE}

For a material in thermodynamic equilibrium the OCV is fully determined by the difference in the chemical potential of lithium between the anode and cathode. We estimate the chemical potential of lithium at the titania cathode from the computed variation of the total energy with concentration. In order to compute the chemical potential exactly the energy for all possible configurations of Li ions at each concentration is required. As discussed above the dominant interactions governing the ordering are due to the elastic screening of the $\mathrm{Li}-\mathrm{Li}$ repulsions. As these effective interactions vary strongly with concentration (actually switching from repulsive to attractive) they cannot be described using concentration-independent interaction parameters. Though in principle an empirical potential could be created to describe interactions in this system, even the most sophisticated potential models currently used have difficulty representing pure bulk $\mathrm{TiO}_{2}$ and its surfaces. In particular, to our best knowledge, it has not been possible to produce a model that similtaneously describes both the dielectric and elastic/ vibrational properties. ${ }^{34}$ It would be difficult to create such a potential to describe correctly the concentration dependence of the interactions. A variable charge model of some kind would need to be used, which could reproduce correctly the accommodation of the charge by the lattice. Even if such a potential model could be created it would not be easily used in Monte Carlo calculations of the free energy, which, to date, to our best knowledge have been confined to lattice Monte Carlo simulations in the grand canonical ensemble and thus depend on the concentration independence of the interaction parameters. ${ }^{35}$ The calculation of the chemical potential for the system in question is therefore not possible within a simple lattice Monte Carlo scheme. Instead, the excess free energy is approximated here by numeric differentiation of the total energy (referred to metallic lithium and bulk rutile) with respect to $x$ using the lowest-energy configurations discussed above. This approach has the advantage of very accurate energetics but suffers from the fact that the statistical sampling of low-energy structures is limited. The computed OCV profile is displayed in Fig. 8. Experimentally observed OCV decays monotonically at room temperature, as there is no significant intercalation of Li into the lattice. As elevated temperature, intercalation proceeds and the first discharge after loading has steps at $x \approx \frac{1}{8}$ [of 0.3 (Ref. 9) or $0.1 \mathrm{eV}$ (Ref. 8) ] and at $x \approx \frac{1}{2}$ (of $0.1 \mathrm{eV}$ ). The majority of the discharge capacity occurs on the plateau between $x=\frac{1}{8}$ and $\frac{1}{2}$. These observations are qualitatively similar to the computed OCV. The computed voltage of the plateau is $1.75 \mathrm{eV}$ (note that if lattice relaxation is not allowed this is reduced to $\approx 1.3 \mathrm{eV}$ ), which may be compared to the observed values of 1.4 and $1.5 \mathrm{eV}^{9,8}$

The shape of the OCV curve has been explained previously in terms of a dielectric screening model. ${ }^{8}$ The steps in the OCV were related to ordered structures resulting from filled channels in the $c$ direction spaced in the $a b$ plane. ${ }^{8}$ However, diffusion in rutile is highly anisotropic and abnormally fast along $c .^{36}$ In light of this one would expect structures involving occupancy of the $c$ channels to be accessible

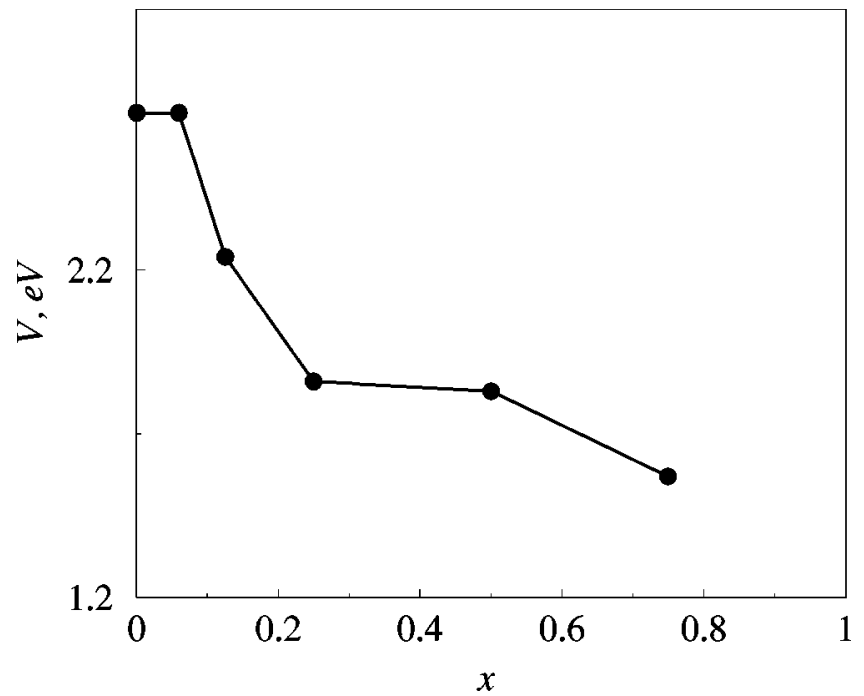

FIG. 8. The calculated open circuit voltage for $\mathrm{Li}_{x} \mathrm{TiO}_{2}$ rutile.

at all temperatures and therefore this model fails to explain the absence of intercalation along $c$ at room temperature. , $38^{3}$ The interpretation of the OCV resulting from the current work is very different. We find that structures with ions arranged along the $c$ channels have energies higher than those of the $a b$-layered structures [Figs. 4(a), 4(b), and 4(d)] by $\approx 0.3 \mathrm{eV} / \mathrm{Li}$ at $x=\frac{1}{8}$ and by $\approx 0.07 \mathrm{eV} / \mathrm{Li}$ at $x=\frac{1}{2}$. The OCV profile can be reinterpreted in the terms of the structures and energies identified here. The initial plateau at $x \leqslant \frac{1}{8}$ (Fig. 8) corresponds to the coexistence of effectively noninteracting $\mathrm{Li}$ ions in $a b$ planes. The plateau between $x=\frac{1}{8}$ and $\frac{1}{2}$ is due to the gradual increase in the occupancy of the $a b$ planes until at $x=\frac{1}{2}$ all sites in the plane are full, resulting in a structure containing lithiated $a b$ planes separated in the $c$ direction. The very efficient and local elastic screening mechanism discussed above yields a linear increase in the total energy for $\frac{1}{8}<x<\frac{1}{2}$, producing the flat plateau in the chemical potential. As the concentration of Li ions increases the screening becomes less efficient until at $x=\frac{1}{2}$ it is no longer possible to avoid the occupancy of nearest-neighbor sites in the $c$ direction. At this point the effective interactions in the $a b$ and $c$ directions are of a similar magnitude. This results in a small step in the OCV at $x=\frac{1}{2}$ when nearestneighbor occupancy in the $c$ direction begins. This explanation reproduces the shape of the experimental discharge curve accurately, but the absolute values computed here are different from those observed. In particular the computed voltage drop between the first and second plateaus is 0.75 rather than the 0.3 (Ref. 9) or $\approx 0.1 \mathrm{eV}$ (Ref. 8) observed. However, the variation in the measured values indicates a strong dependence on experimental conditions. The values computed here rely on absolute thermal equilibrium and assume that Li ions can always access the lowest-energy sites. It seems likely that kinetic restrictions prevent access to the thermodynamically most stable structures studied here. This possibility is now examined in detail.

\section{DIFFUSION}

Although it is clear that Li intercalation is thermodynamically favorable, the observed absence of intercalation at 


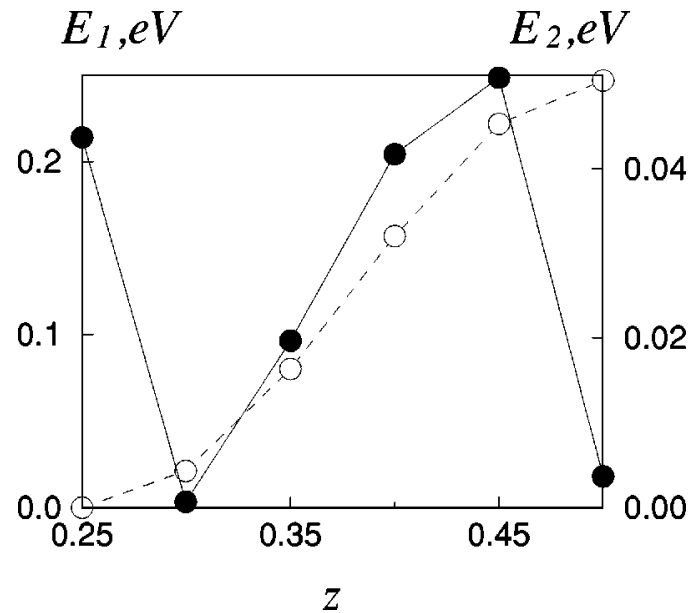

FIG. 9. The relative energy of $\mathrm{Li}_{1 / 2} \mathrm{TiO}_{2}$ as a function of $\mathrm{Li}$-ion position. The open circles mark a structure with a fixed lattice $\left(E_{1}\right.$, left-hand scale) and the filled circles correspond to a fully relaxed structure $\left(E_{2}\right.$, right-hand scale).

room temperature indicates that kinetic restrictions are important. In order to examine Li mobility, adiabatic energy surfaces for Li diffusion have been computed for $x=\frac{1}{2}$ in a single rutile unit cell.

Mobility in the $c$ direction was examined by placing $\mathrm{Li}$ atoms in different positions along the symmetry-unique segment $(0.25 c<z<0.5 c)$ of the line $(0.5,0, z)$. At each position the energy was evaluated for the fixed lattice and also after full relaxation of the internal and cell coordinates. The energy surface, with respect to the minimum, is shown in Fig. 9: $E_{1}$ corresponds to the case of the fixed lattice, and $E_{2}$ to the fully relaxed structure. For a rigid host lattice the energy profile has a maximum at $z=0.5 c$ and a minimum at $0.25 c$. A symmetry-related minimum is located at $z=0.75 c$ and a maximum at 0 . The barrier between the minima is 0.3 $\mathrm{eV}$. When the lattice is allowed to relax the depth of the minimum decreases by $0.15 \mathrm{eV}$ and the position of the minimum shifts to $z=0.30 c$, generating the minimum-energy structure discussed above (Sec. III). In addition, a new minimum of approximately the same depth appears at $z=0.5 c$. The height of the barrier is dramatically decreased to 0.04 $\mathrm{eV}$, facilitating high mobility in the $c$ direction.

In the $a b$ plane the preferred pathway for diffusion is through the tetrahedral site at $(0.25 a, 0.3 b, 0.5 c)$. The barrier for the diffusion is computed to be $\approx 0.8 \mathrm{eV}$ (Fig. 10), which is much higher than that in the $c$ direction. Assuming a typical value for the jump frequency of $10^{12} \mathrm{~s}^{-1}$, and using the above values for the barriers, the diffusion coefficients are computed to be $D_{c}=10^{-6}$ and $D_{a b}=10^{-14} \mathrm{~cm}^{2} / \mathrm{s}$. The experimentally measured value of $D=10^{-6} \mathrm{~cm}^{2} / \mathrm{s}$ (Ref. 36) corresponds to the highest rate of diffusion, and is in excellent agreement with the computed $D_{c}$. This data coupled with the strong elastic screening discussed above suggests a simple model of the temperature dependence of intercalation in rutile.

$\mathrm{Li}$ insertion in rutile is observed to be strongly anisotropic (one dimensional) and proceeds through channels along the $c$ direction. ${ }^{37}$ At low temperature the very slow diffusion in the

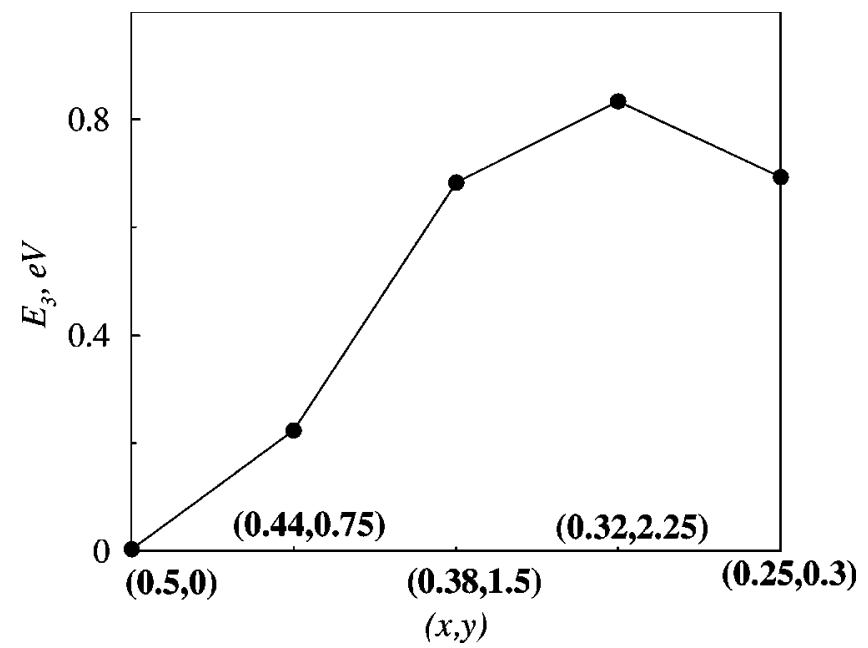

FIG. 10. The change in the total energy as a Li ion is displaced from the octahedral site $(z=0.5 c)$ to a tetrahedral site in the $a b$ plane with full relaxation of the lattice.

$a b$ planes prevents ions from reaching thermodynamically favorable positions. The relatively poor screening of the $\mathrm{Li}-\mathrm{Li}$ interactions in the $c$ direction leads to rather long-range effective repulsive interactions in $c$. At the same time the rapid elastic screening of interactions in the $a b$ plane provides a trapping mechanism for $\mathrm{Li}$-ion pairs (and possibly for larger clusters). Once a pair has formed the $c$ channel is effectively blocked for further intercalation as diffusion in the $a b$ plane is poor. Thus there is no significant intercalation into rutile at ambient temperatures even in the ideal electrode orientation $(001){ }^{6,8,38,39,40}$

At elevated temperatures in-plane diffusion is considerably enhanced, unblocking the $c$ channels and intercalation proceeds. In fact at $120^{\circ} \mathrm{C} \mathrm{Li}$ is observed to intercalate up to full loading. ${ }^{9}$

\section{CHARGE TRANSFER AND ELECTRONIC STRUCTURE}

The mechanisms that underly the thermodynamics and kinetics of $\mathrm{Li}$ intercalation described above are dominated by the strong coupling between the charge, structural, and spin degrees of freedom. In this section the details of charge and spin distributions are examined and the associated electronic structure discussed. A Mulliken population analysis is used to monitor ionic charges and spin states and also to define ion-projected partial densities of states. The details of the changes in charge, spin, and local electronic structure may be accessible to spectroscopic, spin-resonance, or electronenergy-loss spectroscopy experiments, which might provide further insight into the mechanisms discussed in the current work.

In order to separate the structural and electronic contributions to the distribution of electrons donated to the lattice we have computed charge and spin densities in the lithiated structures discussed above and in similar structures from which the Li ions and the donated electrons have been removed without relaxing the lattice (in what follows these are referred to as delithiated structures). In order to discuss the 


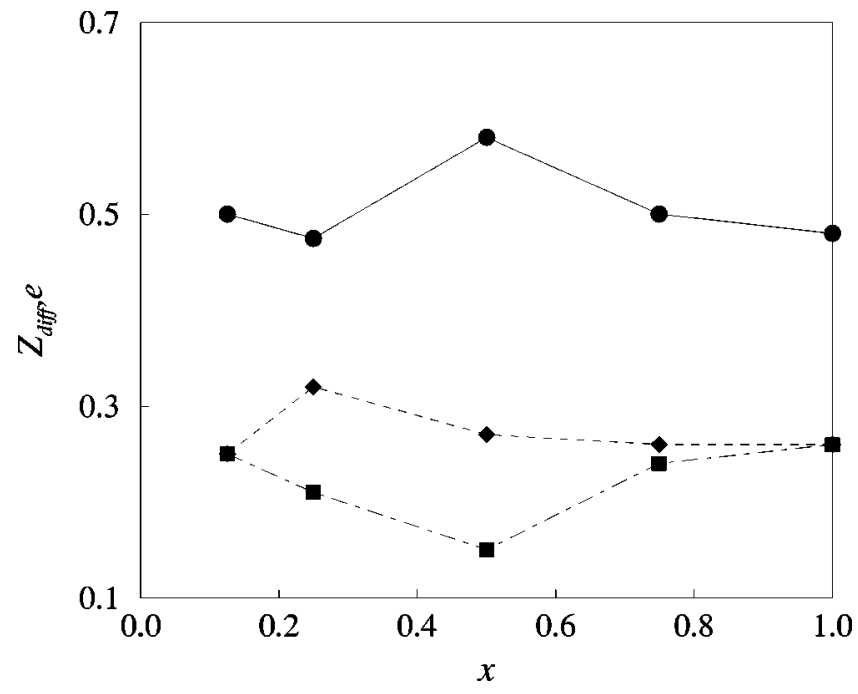

FIG. 11. The difference in charge of the Ti (solid line) and $\mathrm{O}$ ions (dashed line), and the charge retained by the $\mathrm{Li}$ ions (dotdashed line) in the lithiated and delithiated structures.

electron donated to the lattice on $\mathrm{Li}$ insertion we consider it to be separated into induced changes in the charge of the Ti and $\mathrm{O}$ ions and that retained by the $\mathrm{Li}$ ion. These contributions sum to one electron per donated $\mathrm{Li}$ ion. The difference between the charge distributions in the lithiated and delithiated structures is plotted with respect to Li-ion concentration in Fig. 11. In general, approximately half of the additional electron is transferred to the Ti ions $\left(60 \%\right.$ at $\left.x=\frac{1}{2}\right), 25 \%-$ $30 \%$ to the $\mathrm{O}$ ions, and $15 \%-25 \%$ retained by the $\mathrm{Li}$ ions with the distribution depending some what on the Li-ion concentration. As the $a b$ planes are filled the charge retained by the $\mathrm{Li}$ ions decreases until the planes are "full" at $x=\frac{1}{2}$ with the majority of this charge being transferred to the $\mathrm{Ti}$ ions.

The charge and spin densities donated to the lattice are not homogeneously distributed. Remarkably, the majority of the charge localizes on $\mathrm{Ti}$ sites neighboring the $\mathrm{Li}$ ion but the spin is localized on the second-nearest-neighbor Ti ions. The charge on the Ti ions receiving the most charge and on those receiving the most spin (least charge) and the average charge on the lattice are plotted as a function of Li-ion concentration in Fig. 12. The average charge donated to the Ti ions increases linearly. It is clear that for $x<\frac{1}{2}$ the tendency for localization of the charge on nearest-neighbor $\mathrm{Ti}$ ions is to grow. For $x>\frac{1}{2}$, as filling in the $c$ direction begins, the charge on the neighboring $\mathrm{Ti}$ ions stays roughly constant with further charge being accommodated on the Ti ions of the lattice (Fig. 12). The trends in the variation of the donated spin density are similar; the spin-charge separation decreases rapidly for $x>\frac{1}{2}$ as the charge delocalizes on the lattice. The spin density for $x=\frac{1}{8}$ is depicted in Fig. 13. The unpaired electrons are contained in $d$ orbitals that are oriented in such a way as to minimize their overlaps with the neighboring oxygen ions, minimizing the electrostatic repulsion.

To the authors knowledge, this is the first observation of so-called spin-charge separation in first-principles calculations although the concept has become an important component of recent work on high- $T_{c}$ superconductivity. ${ }^{41-43}$ An

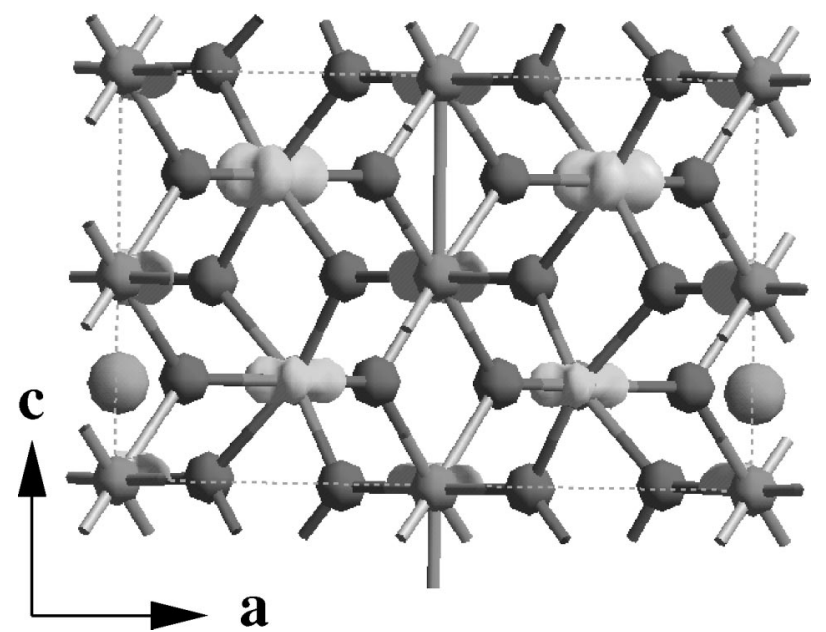

FIG. 12. The variation in charge on the Ti ions receiving most of the charge (dashed line) and most of the spin (solid line) with respect to pure rutile as a function of Li-ion concentration. Note that the average value (dotted line) is growing linearly.

accurate description of spin-charge excitations is beyond the single-particle theory used here. However, DFT is, in principle, capable of describing accurately the spin and the charge densities of the ground state. The details of the separation may depend on the treatment of exchange and correlation effects. ${ }^{44}$ This sensitivity has been examined by repeating the calculations with a hybrid functional [B3LYP (Ref. 45)], which reproduced the same pattern of the spin and charge distributions.

At low $\mathrm{Li}$ concentrations the donated charge density is also localized on $d$ orbitals of the Ti ions. As the ions are well separated in the $a b$ planes one does not expect a direct contribution from these localized "donor" states to electrical conductivity. The conductivity of bulk rutile is highly anisotropic; the electron mobility in the $a b$ plane is almost twice that in the $c$ direction. ${ }^{22}$ Upon $\mathrm{Li}$ intercalation the overall conductivity rises sharply at $x \sim \frac{1}{8} \cdot{ }^{9}$ A possible explanation

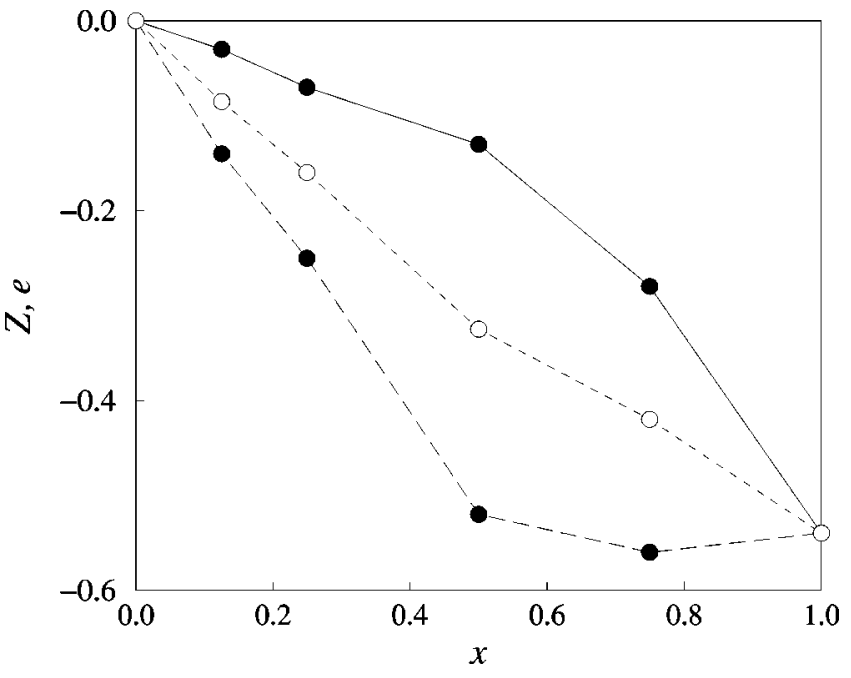

FIG. 13. An isovalue surface of the spin density for $\mathrm{Li}_{1 / 8} \mathrm{TiO}_{2}$ 


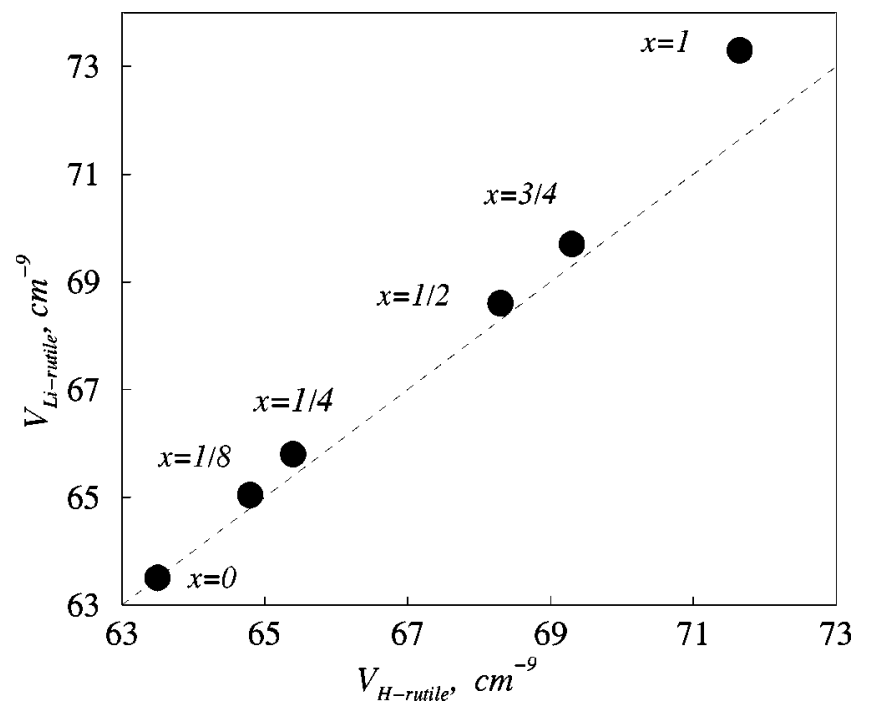

FIG. 14. The volume expansion of similar lithiated and hydrated structures for $x \leqslant 1$. Dashed line $x=y$ is a guide for the eye.

for this behavior emerging from the current study is that the induced structural changes enable conduction in the $c$ direction. As discussed above, as intercalation proceeds, the volume of the unit cell increases but, due to the ordering of the ions in the $a b$ planes, this is achieved through an increase in the $a$ - and $b$-lattice constants while $c$ decreases. The contraction of $c$ is due to the expansion and distortion of the Lioccupied octahedra. As a result there is a close Ti-Ti contact along $c$ between Li-neighboring Ti ions (upon which the donated charge has localized) and the second-neighbor Ti ions. These contacts of $2.75-2.88 \AA$ have been classified previously as intermediate strength Ti-Ti "bonds," 46 and are depicted as such in Fig. 7. It seems likely that the enhanced conductivity in the $c$ direction is mediated by these close Ti-Ti contacts.

The structural distortions may be rationalized in terms of variations in the ionic radii of the $\mathrm{Ti}$ and $\mathrm{O}$ ions rather than the local deformations required to accommodate the intercalated $\mathrm{Li}$ ions. The charge donated to the $\mathrm{O}$ sublattice is localized on the Li-neighboring $\mathrm{O}$ ions. The value of the extra charge per oxygen ion is four times smaller than that per titanium because of the larger number of the nearest oxygens and thus the variation in the effective ionic radius of the $\mathrm{O}$ ions is less than that of the $\mathrm{Ti}$ ions. This leads one to the conclusion that the driving force for structural distortion is predominantly due to the large increase in radius of the $\mathrm{Ti}$ ions. ${ }^{23}$ We have further tested this hypothesis by substituting $\mathrm{H}$ for $\mathrm{Li}$ in the lowest-energy $\mathrm{Li}_{x} \mathrm{TiO}_{2}$ configurations discussed above and fully relaxing the resultant structure. We note that this procedure does not generate the lowest-energy hydrogenated structures, ${ }^{47}$ but $\mathrm{H}$ analogs of the lithiated structures. The volume of hydrogenated structures does not differ significantly from that of the corresponding lithiated structures as shown in Fig. 14. This confirms that volume expansion is largely due to the charge donated to the lattice and thus does not depend significantly on the nature of the inserted ion or on the local deformations required to accommodate it-which are significantly different for $\mathrm{Li}$ and $\mathrm{H}$.
One would expect the reduction of the Ti ions, localization of the donated charge, and charge-spin separation to have major consequences for the electronic structure of lithiated rutile. The band structures of intercalated transitionmetal oxides have been studied extensively with electronic structure calculations. Often the variations induced by doping are analyzed in terms of the rigid-band model, which is based on the assumption that the electronic structure of the host material suffers only minor perturbations during intercalation. In this case electrons donated to the lattice result in a gradual rise of the Fermi level in a fixed band structure. A small modification of the model accounts for the gradual lowering of the band energies due to the Li-ion potential. Previous calculations have demonstrated that the rigid-band model is valid for the $\mathrm{Li}_{1 / 2} \mathrm{TiO}_{2}$ spinel but not for the $\mathrm{LiTiO}_{2}$ hexagonal phase. ${ }^{10}$ Structural deformations induced by intercalation are the main reason for the limited validity of the model. Changes in bond length, and thus effective overlap integrals, have been demonstrated to produce significant shifts in the valence bands for a number of $\mathrm{Li}$ transitionmetal oxides that adopt the hexagonal structure. Relaxation of the lithiated hexagonal phase also induces rearrangements at the bottom of the conduction band. ${ }^{10}$ As discussed above, strong local deformations play a crucial role in the intercalation of $\mathrm{Li}$ into rutile and therefore the validity of the rigidband model is doubtful.

The influence of $\mathrm{Li}$ insertion on the electronic structure of the host is revealed in the variation of the computed density of states (DOS) with concentration. As an example, the DOS for the $\mathrm{Li}_{1 / 2} \mathrm{TiO}_{2}$ rutile-layered structure is shown in Fig. 15. The lowest-energy solution for the relaxed lattice structure is ferromagnetically coupled with $\mathrm{Ti}$ moments of 0.25 and $0.33 \mu_{B}$. The Ti semicore $(3 s$ and $3 p)$ majority-spin $(\alpha)$ bands are shifted to slightly lower energies than the minority-spin $(\beta)$ bands due to the additional exchange interaction with the valence spin density. A remarkable result is that there is essentially no splitting of the Ti semicore peaks due to the different local environments, charges, and spin states of the $\mathrm{Ti}$ ions. For instance, there is no significant splitting of the Ti-3p peak at $x=\frac{1}{2}$ (Fig. 15, lower panel) where two distinct types of $\mathrm{Ti}$ are present in the cell. The effect of structural distortion on the Ti-3p [Ti $(3 s)]$ splitting can be isolated from that of the donated charge by examining the DOS of the delithiated structure. It can be seen from Fig. 15 , upper panel, that the splitting of the Ti-3 $p$ peak due to the local structural distortions is about $1 \mathrm{eV}$. The absence of significant splitting of the semicore states on both the Ti and $\mathrm{O}$ sites for the lithiated structures (which occurs at all the concentrations studied here) appears to be due to a near cancellation of the changes of the local potential due to the extra valence charge and the local structural distortions.

As intercalation proceeds and the charge on the Ti sites rises the semicore bands shift to higher energies relative to the top of the O- $2 p$ valence band as shown in Fig. 16 for the Ti-3s states. For $x>\frac{1}{8}$ the shift is found to be linear in $x$ and is clearly related simply to the average $\mathrm{Ti}$ charge state. The short plateau between $x=\frac{1}{16}$ and $\frac{1}{8}$ coincides with the high screening of $\mathrm{Li}$ interactions discussed in detail in Sec. III. The computed variation in the Ti- $3 p$ level is very similar. 


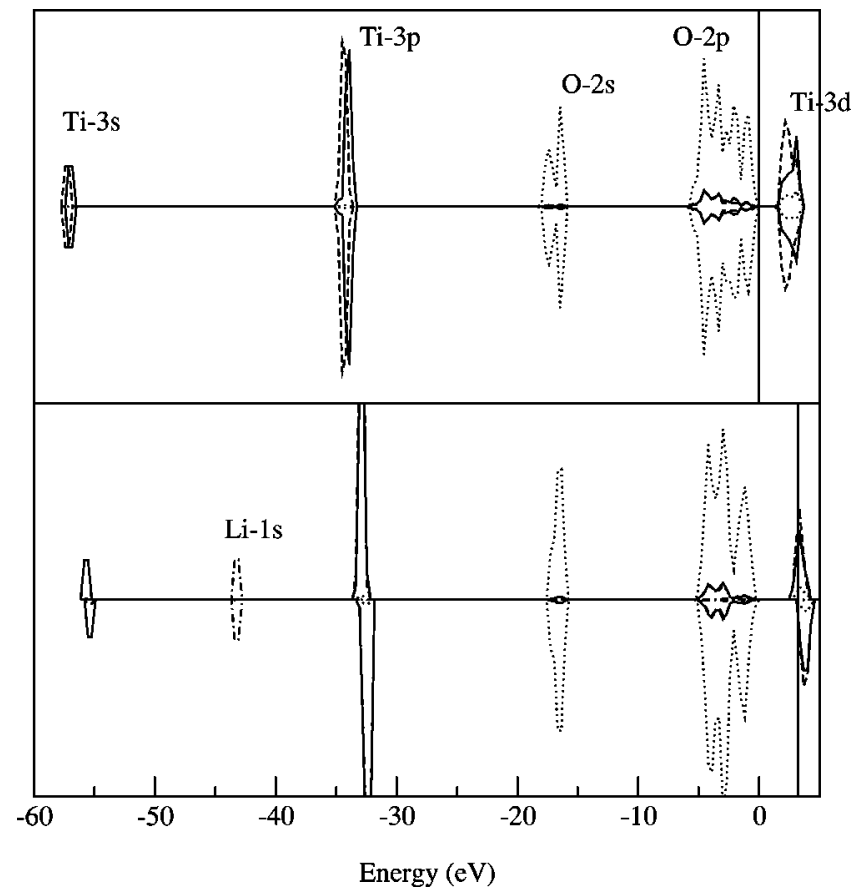

FIG. 15. The atom-projected density of states for $\mathrm{Li}_{1 / 2} \mathrm{TiO}_{2}$ with contributions from majority-spin $(\alpha)$ bands plotted as positive and minority-spin $(\beta)$ bands as negative values. The atomic contributions are $\mathrm{Ti}$ (continuous and dashed lines), $\mathrm{O}$ (dotted line), and $\mathrm{Li}$ (dot-dashed line). The data for the lithiated (lower panel) and delithiated (upper panel) structures are aligned at the valence-band maximum. The Fermi level for each structure is indicated by a vertical line.

One consequence of the strong shift in the semicore levels is that their treatment as core levels in a pseudopotential calculation is unlikely to be an accurate approximation. We have explored this point in some detail and find that if the Ti- $3 s$ and $-3 p$ levels are included in the pseudopotential the inter-

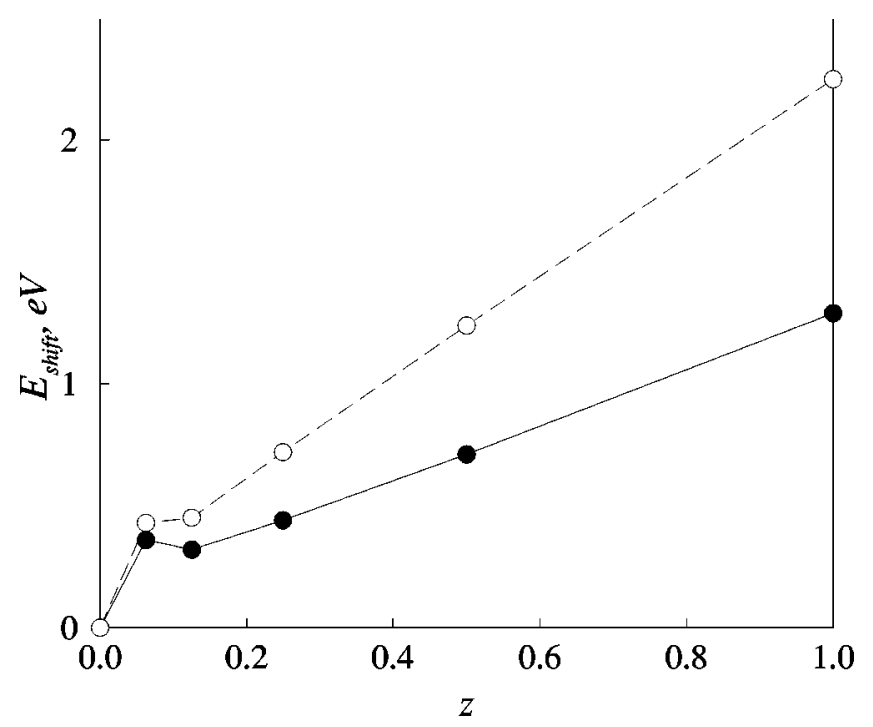

FIG. 16. The variation of the core-level energy, relative to the valence-band maximum, with $\mathrm{Li}$ concentration for the $\mathrm{Ti}(3 s) \alpha$ (solid line) and $\beta$ (dashed line) states.

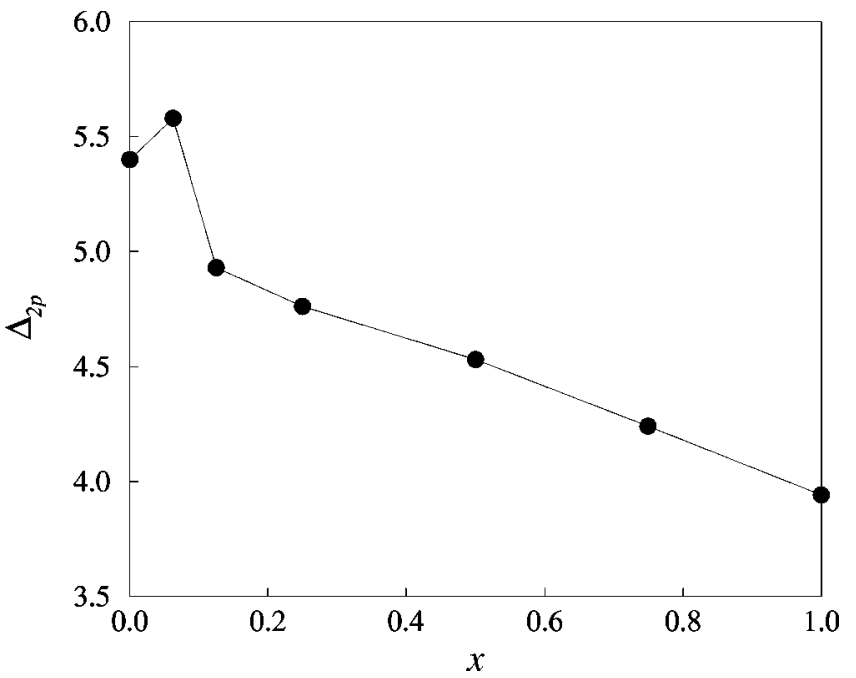

FIG. 17. The variation of the O- $2 p$ bandwidth with Li-ion concentration.

calation energy increases by $0.32 \mathrm{eV}$ per Li at $x=\frac{1}{8}$ and 0.78 $\mathrm{eV}$ per $\mathrm{Li}$ at $x=\frac{1}{4}$. The discrepancy is thus not only significant but also strongly dependent on concentration and leads to large errors in the computed OCV.

The lower edge of the conduction band consists mainly of Ti-3 $d$ states with a small admixture of O-2p. The upper valence band consists predominantly of the $\mathrm{O}-2 p$ derived states with a small admixture of $\mathrm{Ti}$ and $\mathrm{Li}$ states. The small participation of Li states increases with Li content. The upper valence band of pure rutile consists of a two-peak structure, which, on lithium insertion, gradually changes to the threepeak structure seen for $\mathrm{Li}_{1 / 2} \mathrm{TiO}_{2}$ in Fig. 15. The additional structure is related to the local lattice distortions that disturb the near octahedral symmetry of rutile.

Another clear trend is the narrowing of the O- $2 p$ band due to Li-induced volume expansion, which reduces the ef-

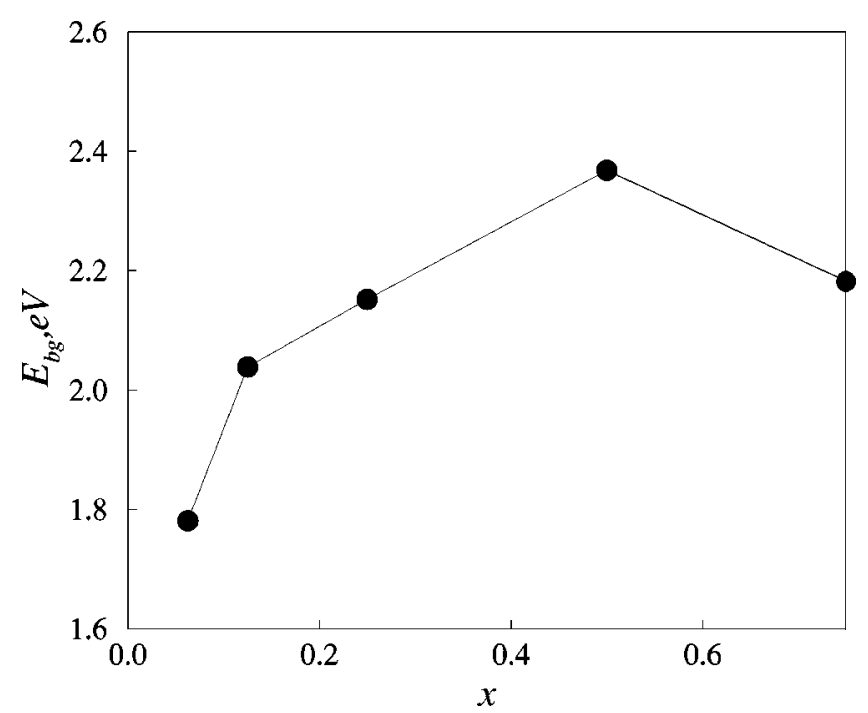

FIG. 18. The variation in the energy difference between the valence-band maximum and conduction-band minimum with lithium concentration. 
fective overlap of the O- $2 p$ and Ti- $3 d$ orbitals (Fig. 17). The computed bandwidth decreased from $5.4 \mathrm{eV}$ for pure rutile [experimental value $5.4 \mathrm{eV}$ (Ref. 48)] to $4.55 \mathrm{eV}$ at $x=\frac{1}{2}$. The variation of the band gap with Li concentration is displayed in Fig. 18. The band gap of pure rutile is computed to be $\approx 2 \mathrm{eV}$ while the observed value is approximately $3 \mathrm{eV}^{49}$ This discrepancy is due to the well-known tendency of DFT theory to underestimate the absolute magnitude of the band gap. The overall trend for the gap to open as $\mathrm{Li}$ is intercalated is predominantly due to the narrowing of the $\mathrm{O}-2 p$ band.

\section{CONCLUSIONS}

The details of Li intercalation into rutile have been established by extensive density-functional simulations. Li-ion site preferences and the thermodynamically most stable configurations are strongly dependent on Li-ion concentration. At all concentrations the Li-intercalated lattice is found to be stable with respect to rutile and metallic lithium. The effective Li-Li interactions are highly anisotropic and concentration dependent and so are not easily amenable to parametri- zation for lattice Monte Carlo simulations. The screening of these interactions is achieved by local elastic distortions that are closely related to soft vibrational modes. This mechanism leads to configurations that consist of $\mathrm{Li}$ ions ordered in the $a b$ planes rather than in the $c$ direction as predicted by the previously accepted dielectric screening model. The elastic screening is very efficient and at $x=\frac{1}{8}$ the ions are essentially noninteracting. The anisotropic expansion of the volume is best interpreted as being due to the increase in radii of reduced $\mathrm{Ti}$ ions rather than the accommodation of $\mathrm{Li}^{+}$ions in the lattice.

The computed Li-ion diffusion constants are very anisotropic and in excellent agreement with those observed. Fast diffusion in the $c$ direction is seen to originate from the elastic screening mechanism and anisotropic volume expansion. A model of Li-ion intercalation has been proposed, taking into account both thermodynamic and kinetic factors that resolve apparent contradictions in the interpretation of the temperature dependence of OCV measurements. Changes in the electronic structure and their relationship to the interaction between structural, charge, and spin degrees of freedom have been discussed in detail.
${ }^{1}$ D. O'Hare, in Inorganic Materials, edited by D. W. Bruce and D. O’Hare (Wiley, New York, 1996), pp. 171-254.

${ }^{2}$ S. Huang, L. Kavan, A. Kay, and M. Grätzel, J. Electrochem. Soc. 141, 142 (1995).

${ }^{3}$ B. O'Regan and M. Grätzel, Nature (London) 353, 737 (1991).

${ }^{4}$ M. Voinov, Proc.-Electrochem. Soc. 81, 352 (1981).

${ }^{5}$ F. Bonino, L. Busani, M. Manstretta, B. Rivolta, and B. Scrosatti, J. Power Sources 14, 261 (1985).

${ }^{6}$ T. Ohzuku, Z. Takehara, and S. Yoshizawa, Electrochim. Acta 27, 1263 (1982).

${ }^{7}$ L. Kavan, D. Fattakhova, and P. Krtil, J. Electrochem. Soc. 146, 1375 (1999).

${ }^{8}$ B. Zachau-Christiansen, K. West, and S. Atlung, Solid State Ionics 28-30, 1176 (1988).

${ }^{9}$ W. J. Macklin and R. J. Neat, Solid State Ionics 53-56, 694 (1992).

${ }^{10}$ L. Benco, J. L. Barras, and C. A. Daul, Inorg. Chem. 38, 20 (1999).

${ }^{11}$ A. Stashans, S. Lunell, R. Begstrom, A. Hagfedt, and S. E. Lindquist, Phys. Rev. B 53, 159 (1996).

${ }^{12}$ M. K. Aydinol, A. F. Kohan, G. Ceder, K. Cho, and J. Joannopoulos, Phys. Rev. B 56, 1354 (1997).

${ }^{13}$ W. C. Mackrodt, J. Solid State Chem. 142, 428 (1999).

${ }^{14}$ M. V. Koudriachova, N. M. Harrison, and S. W. de Leeuw, Phys. Rev. Lett. 86, 1275 (2001).

${ }^{15}$ C. Lee, P. Ghosez, and X. Gonze, Phys. Rev. B 50, 13379 (1994).

${ }^{16}$ M. C. Payne, M. P. Teter, D. C. Allan, T. A. Arias, and J. D. Joannopoulos, Rev. Mod. Phys. 64, 1045 (1992).

${ }^{17}$ CASTEP 3.9 Academic version, licensed under the UKCP-MSI agreement (1999).

${ }^{18}$ R. Dovesi, V. R. Saunders, C. Roetti, M. Causá, N. M. Harrison, R. Orlando, and C. M. Zicovich-Wilson, computer code
CRYSTAL98, user's manual (University of Torino, Torino, Italy, 1998).

${ }^{19}$ J. P. Perdew, Phys. Rev. B 34, 7406(E) (1986).

${ }^{20}$ D. Vanderbilt, Phys. Rev. B 41, 7892 (1990).

${ }^{21}$ A. T. Paxton and L. Thien-Nga, Phys. Rev. B 57, 1579 (1998).

${ }^{22}$ J. K. Burdett, T. Hughbanks, G. J. Miller, J. W. Richardson, and J. V. Smith, J. Am. Chem. Soc. 109, 3639 (1987).

${ }^{23}$ R. G. Breckenridge and W. R. Hosler, Phys. Rev. 91, 793 (1953).

${ }^{24}$ J. Akimoto, Y. Gotoh, M. Sohma, K. Kawaguchi, and Y. Oosawa, J. Solid State Chem. 110, 150 (1994).

${ }^{25}$ J. G. Traylor, H. G. Smith, R. M. Nicklow, and M. K. Wilkinson, Phys. Rev. B 3, 3457 (1971).

${ }^{26}$ D. M. Eagles, J. Phys. Chem. Solids 25, 1243 (1964).

${ }^{27}$ A. M. Hofmeister, J. Xu, and S. Akimoto, Am. Mineral. 75, 951 (1990).

${ }^{28}$ C. Lee and X. Gonze, Phys. Rev. Lett. 72, 1686 (1994).

${ }^{29}$ S. P. S. Porto, P. A. Fleury, and T. C. Damen, Phys. Rev. 154, 522 (1967).

${ }^{30}$ M. F. Vigasina, E. V. Guseva, and R. Yu. Orlov, Fiz. Tverd. Tela (Leningrad) 31, 47 (1989) [Sov. Phys. Solid State 31, 747 (1989)].

${ }^{31}$ T. A. Hewston and B. L. Chamberland, J. Phys. Chem. Solids 48, 97 (1987).

${ }^{32}$ M. V. Koudriachova, N. M. Harrison, and S. W. de Leeuw (unpublished).

${ }^{33}$ J. Akimoto, Y. Gotoh, K. Kawaguchi, and Y. Oosawa, J. Solid State Chem. 96, 446 (1992).

${ }^{34}$ V. Swamy, J. Muscat, J. D. Gale, and N. M. Harrison, Surf. Sci. 504, 115 (2002).

${ }^{35}$ C. Wolverton and A. Zunger, Phys. Rev. Lett. 81, 606 (1998).

${ }^{36}$ O. W. Johnson, Phys. Rev. A 136, 284 (1964).

${ }^{37}$ H. O. Finklea, in Semiconductor Electrodes, Studies in Physical 
and Theoretical Chemistry (Elsevier, Amsterdam, 1988), Vol. 55, pp. 43-145.

${ }^{38}$ F. Bonino, L. Busani, M. Lazzari, M. Mantretta, and B. Scrosatti, J. Power Sources 6, 261 (1981).

${ }^{39}$ D. W. Murphy, R. J. Cava, S. M. Zahurak, and A. Santoro, Solid State Ionics 9-10, 413 (1983).

${ }^{40}$ D. W. Murphy, P. A. Christensen, J. N. Carides, and F. J. di Salvo, in Fast Ion Transport in Solids (North-Holland, Amsterdam, 1979), p. 137.

${ }^{41}$ P. W. Anderson, Science 235, 1196 (1987).

${ }^{42}$ S. Kivelson, D. S. Rokhsar, and J. Sethna, Phys. Rev. B 35, 8865 (1987).
${ }^{43}$ T. Senthil and M. P. A. Fisher, cond-mat/006500 (unpublished).

${ }^{44}$ J. Muscat, N. M. Harrison, and G. Thornton, Phys. Rev. B 59, 15457 (1999).

${ }^{45}$ A. D. Becke, J. Chem. Phys. 98, 5648 (1993).

${ }^{46}$ S. Asbrink and A. Magneli, Acta Crystallogr. 12, 575 (1959).

${ }^{47}$ M. V. Koudriachova, N. M. Harrison, and S. W. de Leeuw (unpublished).

${ }^{48}$ S. P. Kowlczyk, F. R. McFeely, L. Ley, V. T. Gritsyn, and D. Schirley, Solid State Commun. 23, 161 (1977).

${ }^{49}$ J. Pascual, J. Camassel, and H. Mathieu, Phys. Rev. B 18, 5606 (1978). 